UT-11-17

May, 2011

\title{
SUSY CP Problem in Gauge Mediation Model
}

\author{
Takeo Moroi and Norimi Yokozaki \\ Department of Physics, University of Tokyo, Tokyo 113-0033, JAPAN
}

\begin{abstract}
SUSY CP problem in the gauge mediation supersymmetry breaking model is reconsidered. We pay particular attention to two sources of CP violating phases whose effects were not seriously studied before; one is the effect of the breaking of the GUT relation among the gaugino masses due to the field responsible for the GUT symmetry breaking, and the other is the supergravity effect on the supersymmetry breaking parameters, in particular, on the bi-linear supersymmetry breaking Higgs mass term. We show that both of them can induce too large electric dipole moments of electron, neutron, and so on, to be consistent with the experimental bounds.
\end{abstract}




\section{Introduction}

Low-energy supersymmetry (SUSY), if it exists, affects various low-energy phenomenology. The most prominent place to look for the effects of SUSY particles is the LHC experiment because it can provide a direct confirmation of the existence of SUSY particles. However, precision experiments, in particular, those related to flavor and/or CP violation, also put severe constraints on supersymmetric models. The masses of superparticles are required to be larger than $10-100 \mathrm{TeV}$ to avoid flavor and $\mathrm{CP}$ constraints if there is no mechanism to suppress off-diagonal elements of the sfermion mass matrices and $\mathrm{CP}$ violating phases [1]. Such a large value of the superparticle masses are unacceptable from the naturalness point of view.

One of the attractive mechanisms to avoid the SUSY flavor problem is the gauge mediation [2, 3, 4],\#1 In the gauge mediation model, sfermion masses are generated via the interaction with gauge multiplet, so the degeneracy of the masses of sfermions which have same gauge quantum numbers is guaranteed. In such a framework, however, it is still nontrivial to avoid the SUSY CP problem. This is because, even in the gauge mediation model, new $\mathrm{CP}$ violating phases arise in operators which are flavor blind. In particular, relative phases among the gaugino masses, SUSY invariant Higgs mass parameter, and the soft SUSY breaking bi-linear Higgs mass (i.e., so-called $B_{\mu}$ parameter) do not vanish in general, which often results in too large electric dipole moments (EDMs) of electron, neutron, and so on [10]. As in other cases, the SUSY CP problem is serious in the gauge mediation SUSY breaking scenario. So far, a possibility to solve the SUSY CP problem in the gauge mediation model is thought to consider a model in which the $B_{\mu}$ parameter vanishes at the messenger scale [11, 12].

In this Letter, we reconsider the SUSY CP problem. We will concentrate on the effects of $\mathrm{CP}$ violating phases which have not been seriously considered before; we study two important effects which are from (i) the breaking of the grand-unified-theory (GUT) relation among the gaugino masses due to the GUT symmetry breaking field, and (ii) the supergravity contribution to the $B_{\mu}$ parameter. As we see in the following, these possibly become sources of too large EDMs to be consistent with the current constraints. Thus, even if one adopts a model with vanishing $B_{\mu}$ parameter at the messenger scale $M_{\text {mess }}$, which has been regarded as a solution to the SUSY CP problem, too large EDMs may be still induced.

\section{SUSY CP Problem in GMSB}

First, let us give an overview of the $\mathrm{CP}$ violating phases which are relevant to our study. In the present study, the most important $\mathrm{CP}$ violating phases are relative phases among the gaugino masses $M_{A}$, the SUSY invariant Higgs mass $\mu_{H}$, and the soft-SUSY breaking Higgs mass parameter $B_{\mu}$. (In the following, $A=1,2$, and 3 correspond to $U(1)_{\mathrm{Y}}, S U(2)_{\mathrm{L}}$, and $S U(3)_{\mathrm{C}}$, respectively.)

${ }^{\# 1}$ For early attempts, see also [5, 6, 7, 8, 9]. 
To see this, we denote the relevant part of the soft SUSY breaking terms as

$$
\mathcal{L}_{\text {soft }}=-\frac{1}{2} \sum_{A} M_{A} \lambda_{A} \lambda_{A}+B_{\mu} \mu_{H} H_{u} H_{d}+\text { h.c. },
$$

where $\lambda_{A}$ is the gaugino field, while $H_{u}$ and $H_{d}$ are up- and down-type Higgs boson, respectively. In the minimal supersymmetric standard model (MSSM), the following phases are invariant under the phase rotation of the MSSM fields and hence are physical:\#2

$$
\phi_{A}=\arg \left(M_{A} B_{\mu}^{*}\right) .
$$

If these phases are non-vanishing, they become the sources of EDMs (and other CP violating quantities). Thus, those phases are stringently constrained although, in many models, they are expected to be of $O(1)$. In order to solve the SUSY CP problem, it is inevitable to make all the phases of the gaugino masses equal; otherwise, it is impossible to realize $\phi_{1}=\phi_{2}=$ $\phi_{3}=0$. In the simplest model of GMSB, this is the case because the gaugino masses obey the GUT relation.

One possibility of solving the SUSY CP problem is to adopt a model in which the $B_{\mu}$ parameter vanishes at the messenger scale [11, 12. In the gauge mediation model, the scalar tri-linear coupling constants at the messenger scale are expected to vanish (at least at the one-loop level). Thus, we can rotate away all the $\mathrm{CP}$ violating phases (other than the CKM phase) from the MSSM Lagrangian if all the phases in the gaugino masses are aligned. Assuming that the low-energy effective theory below the messenger scale is the MSSM, the $B_{\mu}$ parameter obeys the following renormalization group equation

$$
\frac{d B_{\mu}}{d \ln \mu}=\frac{1}{8 \pi^{2}}\left[3 g_{2}^{2} M_{2}+g_{1}^{2} M_{1}-3 \operatorname{tr}\left(Y_{U}^{\dagger} A_{U}\right)-3 \operatorname{tr}\left(Y_{D}^{\dagger} A_{D}\right)-\operatorname{tr}\left(Y_{L}^{\dagger} A_{L}\right)\right]
$$

where $Y_{U}, Y_{D}$, and $Y_{L}$, are $3 \times 3$ Yukawa matrices for up-type quarks, down-type quarks, and leptons, respectively, while $A_{U}, A_{D}$, and $A_{L}$, are corresponding tri-linear scalar coupling matrices. In addition, $g_{2}$ and $g_{1}$ are gauge coupling constants for $S U(2)_{\mathrm{L}}$, and $U(1)_{\mathrm{Y}}$, respectively. Then, even if $B_{\mu}\left(M_{\text {mess }}\right)=0, B_{\mu}$ at the electroweak scale is generated from the renormalization-group effect, which gives a viable Higgs potential consistent with the electroweak symmetry breaking.

Another possibility is an accidental cancellation among the phases. Even though such a cancellation requires a tuning of underlying parameters, all the phases can be simultaneously made small if the relation $\phi_{1}=\phi_{2}=\phi_{3}$ is realized. This is the case if the GUT relation among the gaugino masses holds.

In the following, we do not specify the mechanism to generate the $\mu_{H}$ and $B_{\mu}$ parameters. Our purpose is to study the effect of the GUT symmetry breaking and the supergravity effect which have not been studied in detail. So we assume that $\phi_{A}$ vanish in the limit that the above mentioned effects are neglected.

\footnotetext{
${ }^{\# 2}$ The effects of the phases in the tri-linear coupling constants (i.e., the so-called $A$ parameters) on EDMs do not have $\tan \beta$ enhancement and are at most of the same order of those of $\phi_{A}$. In our numerical calculation, effects of the phases of $A$ parameters are properly taken into account.
} 


\section{Effect of GUT Relation Breaking}

First, we consider the CP violation from the breaking of the GUT symmetry. In simple models of gauge mediation which neglects the effects of GUT symmetry breaking, it is guaranteed that $\phi_{1}=\phi_{2}=\phi_{3}$.

We start our discussion with a general case; we parameterize the superpotential of the messenger sector as

$$
W=\sum_{Q}\left(\lambda_{i j}^{(Q)} S+M_{i j}^{(Q)}\right) \bar{Q}_{i} Q_{j}
$$

where $Q$ and $\bar{Q}$ denote vector-like chiral multiplets which are in irreducible representations of the standard-model gauge group $G_{\mathrm{SM}}=S U(3)_{\mathrm{C}} \times S U(2)_{\mathrm{L}} \times U(1)_{\mathrm{Y}}$. (The gauge indices are implicit.) Here, $\lambda_{i j}$ is a coupling constant while $M_{i j}$ a mass parameter. In some of gauge mediation models, in particular, those adopting the ISS mechanism of SUSY breaking [13, $M_{i j}$ is non-vanishing [14], although there exist models with $M_{i j}=0$. (For models with $M_{i j} \neq 0$, see also [15, 16].) As we will see, the existence of the mass parameter significantly affects the aspect of CP violation in the SUSY breaking parameters. In general, there may exist several multiplets in the same representation of $G_{\mathrm{SM}}$; those multiplets are distinguished by the indices $i$ and $j$ which run $1-N^{(Q)}$, with $N^{(Q)}$ being the number of $Q$ and $\bar{Q}$ pair. Then, the summation in Eq. (41) is over all the irreducible representation of the $G_{\mathrm{SM}}$. In addition, $S$ is the singlet superfield whose $F$-component is non-vanishing; if there exists several singlet fields, we take their linear combination such that only $S$ acquires $F$-component VEV. It should be understood that $M^{(Q)}$ contains SUSY invariant mass of $Q$ and $\bar{Q}$ arising from the VEV of singlet fields other than $S$.

At the leading order of $F_{S}$, the gaugino mass is given by

$$
m_{A}=\frac{g_{A}^{2}}{16 \pi^{2}} \sum_{Q} b_{A}^{(Q)} \Lambda^{(Q)}
$$

where $b_{A}^{(Q)}$ is the $\beta$-function coefficient of the gauge coupling constant $g_{A}$ due to the chiral multiplet $Q$. In addition, $\Lambda^{(Q)}$ is the ratio between the SUSY breaking mass squared and the supersymmetric mass of the multiplet $Q$ and is given by

$$
\Lambda^{(Q)} \equiv F_{S} \lambda_{i j}^{(Q)}\left[\lambda^{(Q)}\langle S\rangle+M^{(Q)}\right]_{j i}^{-1}
$$

with $\left[\lambda^{(Q)}\langle S\rangle+M^{(Q)}\right]^{-1}$ being the inverse matrix of $\lambda_{i j}^{(Q)}\langle S\rangle+M_{i j}^{(Q)}$.

When there is no supersymmetric mass term (i.e., $M^{(Q)}=0$ ), we can easily see that the phases of all the gaugino masses are aligned; indeed, in such a case, $\Lambda^{(Q)}$ becomes $N^{(Q)} F_{S} /\langle S\rangle$ and is independent of the parameters in superpotential. Notice that this conclusion is independent of the assumption of GUT.

Even if there exists supersymmetric mass term, the phases of gaugino masses become the same as long as the exact GUT relations hold among $\lambda_{i j}^{(Q)}$ and $M_{i j}^{(Q)}$. If $Q$ and $Q^{\prime}$ are 
in a same irreducible representation of GUT group $G_{\mathrm{GUT}}$, the relations $\lambda_{i j}^{(Q)}=\lambda_{i j}^{\left(Q^{\prime}\right)}$ and $M_{i j}^{(Q)}=M_{i j}^{\left(Q^{\prime}\right)}$ are realized with neglecting $G_{\mathrm{GUT}}$ breaking effects. The above relations, however, break down once we take account of the effect of $G_{\mathrm{GUT}}$ breaking.

It is obvious that the relation $\phi_{1}=\phi_{2}=\phi_{3}$ is not guaranteed if the GUT relation $\lambda_{i j}^{(Q)}=\lambda_{i j}^{\left(Q^{\prime}\right)}$ or $M_{i j}^{(Q)}=M_{i j}^{\left(Q^{\prime}\right)}$ is somehow violated for $Q$ and $Q^{\prime}$ from the same multiplet of $G_{\mathrm{GUT}}$. Violation may happen due to operators including the field responsible for the GUT symmetry breaking $G_{\mathrm{GUT}} \rightarrow G_{\mathrm{SM}}$. (We denote such a field $\Sigma$.)

We assume that all the higher-dimensional operators allowed by the $G_{\mathrm{GUT}}$ exist with the cut-off scale of the order of the Planck scale $M_{\mathrm{Pl}} \simeq 2.4 \times 10^{18} \mathrm{GeV}$. Then, their effects are expected to be proportional to powers of $\langle\Sigma\rangle / M_{\mathrm{Pl}}$, where $\langle\cdots\rangle$ denotes vacuum expectation value $(\mathrm{VEV})$. The $\mathrm{VEV}\langle\Sigma\rangle$ is expected to be of the order of the GUT scale $M_{\mathrm{GUT}} \simeq$ $2 \times 10^{16} \mathrm{GeV}$. Importantly, the higher-dimensional operators not only induce the breaking of the GUT relations but also provide a new source of $\mathrm{CP}$ violation because the coefficients of the higher-dimensional operators are complex in general. Even though the effect of the higher-dimensional operator is expected to be suppressed by powers of $M_{\mathrm{GUT}} / M_{\mathrm{Pl}} \sim 10^{-2}$, it can be large enough to be constrained by present and future experiments. In particular, sizable EDMs can be generated, as we see below.

The size of the breaking of the GUT relation is determined by the structure of the superpotential, which depends on the properties of the $G_{\mathrm{GUT}}$ breaking field $\Sigma$ and the vectorlike messenger multiplet. In order to make our discussion concrete and quantitative, we consider the simplest gauge mediation model based on $S U(5)$ SUSY GUT; the messenger multiplet is assumed to consist of $\mathbf{5}$ and $\overline{\mathbf{5}}$ representations of $S U(5)_{\mathrm{GUT}}$, which we denote $\Psi$ and $\bar{\Psi}$, respectively. Notice that $\mathbf{5} \otimes \overline{\mathbf{5}}=\mathbf{1} \oplus \mathbf{2 4}$. Thus, if the GUT symmetry is broken by a field in 24 representation, the effect of GUT breaking can be of the order of $M_{\mathrm{GUT}} / M_{\mathrm{Pl}}$; otherwise, the effect is more suppressed. Below the GUT scale, the vector-like multiplets split into $\psi_{d}, \psi_{l}$ (which are from $\Psi$ ), $\bar{\psi}_{d}$ and $\bar{\psi}_{l}$ (which are from $\bar{\Psi}$ ), whose transformation properties under the standard-model gauge group are $(\mathbf{3}, \mathbf{1},-1 / 3),(\mathbf{1}, \mathbf{2}, 1 / 2),(\overline{\mathbf{3}}, \mathbf{1}, 1 / 3)$, and $(\mathbf{1}, \mathbf{2},-1 / 2)$, respectively.

The first case is that $G_{\mathrm{GUT}}$ is broken by a field which transforms as $\mathbf{2 4}$ of $S U(5)_{\mathrm{GUT}}$, as in the minimal SUSY GUT [17, 18]. We denote the GUT breaking field as $\Sigma_{\beta}^{\alpha}$ (where $\alpha$ and $\beta$ are $S U(5)_{\text {GUT }}$ indices, which run $1-5$ ), whose vacuum-expectation value is parameterized as

$$
\langle\Sigma\rangle=\frac{v_{\mathbf{2 4}}}{2 \sqrt{15}} \operatorname{diag}(2,2,2,-3,-3) .
$$

Then the mass of $X$ - and $Y$-bosons is given by $m_{X, Y}=\sqrt{\frac{5}{6}} g_{5} v_{\mathbf{2 4}}$, where $g_{5}$ is the gauge coupling constant at the GUT scale. In this case, the effects of the breaking of the GUT 
relation is proportional to $v_{\mathbf{2 4}} / M_{\mathrm{Pl}} \# 3$

$$
\begin{aligned}
W & =\lambda_{0} S \bar{\Psi}^{\alpha} \Psi_{\alpha}+\frac{\lambda_{1}}{M_{\mathrm{Pl}}} S \bar{\Psi}^{\alpha} \Sigma_{\alpha}^{\beta} \Psi_{\beta}+M \bar{\Psi}^{\alpha} \Psi_{\alpha} \\
& =\lambda_{0}\left(1+\frac{1}{\sqrt{15}} \epsilon_{\mathbf{2 4}}\right) S \bar{\psi}_{d} \psi_{d}+\lambda_{0}\left(1-\frac{3}{2 \sqrt{15}} \epsilon_{\mathbf{2 4}}\right) S \bar{\psi}_{l} \psi_{l}+\cdots
\end{aligned}
$$

where

$$
\epsilon_{\mathbf{2 4}}=\frac{\lambda_{1} v_{\mathbf{2 4}}}{\lambda_{0} M_{\mathrm{Pl}}}
$$

Then, assuming $\lambda_{0}\langle S\rangle \ll M$, the gaugino masses are given by

$$
M_{A}=\frac{g_{A}^{2}}{16 \pi^{2}}\left(1+\kappa_{A}^{(\mathbf{2 4})} \epsilon_{\mathbf{2 4}}\right) \Lambda
$$

with $\kappa_{1}^{(\mathbf{2 4})}=-\frac{1}{2 \sqrt{15}}, \kappa_{2}^{(\mathbf{2 4})}=-\frac{3}{2 \sqrt{15}}, \kappa_{3}^{(\mathbf{2 4})}=\frac{1}{\sqrt{15}}$, and

$$
\Lambda=\frac{\lambda_{0} F_{S}}{\lambda_{0}\langle S\rangle+M}
$$

Here, we only consider the leading-order contribution from $v_{\mathbf{2 4}}$.

In this framework, we calculate the EDMs of the electron, the neutron and the mercury. As we will see below, since the phase of $B_{\mu}$ is not aligned to those of gaugino masses, the physical phase $\phi_{A}$ become non-negligible, resulting in sizable EDMs. Here, we take $\left|\epsilon_{\mathbf{2 4}}\right|=10^{-2}$, and $\arg \left(\epsilon_{\mathbf{2 4}}\right)=\frac{\pi}{2}$; the phase is chosen to maximize the contribution to the EDMs. With this choice of the parameters, the physical phase $\phi_{A}$ of the order of $O\left(10^{-2}\right)$ is induced, and this phase is large enough to be constrained from the present EDM experiments.

The electron EDM gives a severe constraint on $\phi_{A}$. The experimental bound on the electron EDM is given by [19]

$$
d_{e}<2.1 \times 10^{-27} e \mathrm{~cm} \quad(95 \% \text { C.L. }) .
$$

This bound should be compared to the SUSY contributions, which are dominated by chargino diagrams. The results are shown in panel (a) and (b) of Fig. 1 on $M_{2}$ vs. $\tan \beta$ plane; here, the normalization parameter $\Lambda$ is varied to give the proper value of $M_{2}$, while $\tan \beta$ is calculated as a function of the $B_{\mu}$ parameter at the messenger scale $B_{\mu}\left(M_{\text {mess }}\right)$. In panels (a) and (b), the messenger scales are taken to be $M_{\text {mess }}=10^{6} \mathrm{GeV}$ and $M_{\text {mess }}=10^{12} \mathrm{GeV}$, respectively. One can see that the electron EDM is more enhanced as $\tan \beta$ becomes larger, which is due to the fact that the left-right mixing in the slepton sector is approximately proportional to $\tan \beta$.

${ }^{\# 3}$ GUT symmetry breaking effect can be also on the mass terms, which may induce the EDMs. The sizes of the EDMs are of the same order of those with the GUT symmetry breaking effect on the tri-linear term in the superpotential as far as the correction to the mass term is of the order of $\epsilon_{\mathbf{2 4}} M$. 
One important constraint on the MSSM parameter space may be from the consideration of the anomalous magnetic dipole moment (MDM) of the muon. Recent detailed analysis shows that the deviation between the experimental and theoretical values is at the $3.2 \sigma$ level, which may be due to the physics beyond the standard model [20]. Assuming that the deviation is from the SUSY particles, the SUSY contribution to the muon MDM is [20]

$$
a_{\mu}^{(\mathrm{SUSY})}=(25.9 \pm 8.1) \times 10^{-10} .
$$

In Fig. 1, we also show the region where the muon MDM becomes consistent with the above constraint. One can easily see that, in such a region, the electron EDM becomes one order of magnitude larger than the experimental constraint if $\left|\epsilon_{\mathbf{2 4}}\right|=10^{-2}$ (as far as the phase is of $O(1)$ ).

Hadronic EDMs also give stringent constraints on the size and the phase of $\epsilon_{\mathbf{2 4}}$. Here we consider the neutron EDM and the mercury EDM, induced by the EDMs and chromoelectric dipole moments (CEDMs) of quarks. The experimental bound on the neutron EDM is given by [19]

$$
d_{n}<2.9 \times 10^{-26} e \mathrm{~cm} \quad(95 \% \text { C.L. }),
$$

which should be compared with theoretical estimation [21, 22]\#4

$$
d_{n} \simeq 1.4\left(d_{d}-0.25 d_{u}\right)+1.1 e\left(d_{d}^{c}+0.5 d_{u}^{c}\right),
$$

with $d_{q}$ and $d_{q}^{c}$ being the EDM and CEDM of a quark, respectively. Because the SUSY contributions to the down quark (C)EDM is enhanced by $\tan \beta$, and also because $d_{d}$ and $d_{d}^{c}$ have larger coefficient in Eq.(15), SUSY contribution to $d_{n}$ is dominated by $d_{d}$ and $d_{d}^{c}$ in most of the cases. We have calculated $d_{n}$, and the results are shown in panels (c) and (d) of Fig. 1 with $M_{\text {mess }}=10^{6} \mathrm{GeV}$ and $M_{\text {mess }}=10^{12} \mathrm{GeV}$, respectively. The parameter $\left|\epsilon_{\mathbf{2 4}}\right|=10^{-2}$ is marginally consistent for $M_{\text {mess }}=10^{6} \mathrm{GeV}$, while it is excluded for $M_{\text {mess }}=10^{12} \mathrm{GeV}$ in the parameter region consistent with the muon MDM constraint (13).

The constraint from the mercury EDM is also stringent. The experimental bound is given by [24]

$$
\left|d_{H g}\right|<3.1 \times 10^{-29} e \mathrm{~cm} \quad(95 \% \text { C.L. }),
$$

which can be translated to the upper bound on the CEDMs of quarks as [25, 26]

$$
\tilde{d}_{q} \equiv\left|d_{d}^{c}-d_{u}^{c}\right| \lesssim 4.4 \times 10^{-27} \mathrm{~cm} .
$$

The contours of $\tilde{d}_{q}$ are shown in panels (e) and (f) with $M_{\text {mess }}=10^{6} \mathrm{GeV}$ and $M_{\text {mess }}=$ $10^{12} \mathrm{GeV}$, respectively. The constraint from the mercury EDM is as stringent as the electron EDM constraint.

\footnotetext{
\#4 The contributions from the strange quark are also discussed in the literature [23, and this may be as important as contributions from the up and down quarks. With inclusion of the strange quark contribution, the constraint from the neutron EDM can become as severe as the constraints from the mercury EDM and the electron EDM.
} 
In Fig. 1, the contours of $B_{\mu}\left(M_{\text {mess }}\right)=0$ are also shown, which correspond to vanishing $\phi_{A}$ in the absence of the GUT relation breaking operator. One can see that, adopting the natural value of the $\epsilon_{\mathbf{2 4}}$ parameter, the EDMs become too large to be consistent with the experimental constraints if we adopt (13). Thus, if the breaking of the GUT relation is due to an operator which is linear in $G_{\mathrm{GUT}}$ breaking fields, the SUSY CP problem might remain even in models with vanishing $B_{\mu}$ parameter.

Next, we consider the case that $S U(5)_{\text {GUT }}$ is broken by a field in 75 representation, as in the case of missing partner model [27, 28] $\#$ w5 warameterize the VEV as

$$
\begin{aligned}
\left\langle\Sigma_{c d}^{a b}\right\rangle & =\frac{v_{\mathbf{7 5}}}{4 \sqrt{6}}\left(\delta_{c}^{a} \delta_{d}^{b}-\delta_{d}^{a} \delta_{c}^{b}\right) \\
\langle\Sigma\rangle_{c^{\prime} d^{\prime}}^{a^{\prime} b^{\prime}} & =\frac{3 v_{\mathbf{7 5}}}{4 \sqrt{6}}\left(\delta_{c^{\prime}}^{a^{\prime}} \delta_{d^{\prime}}^{b^{\prime}}-\delta_{d^{\prime}}^{a^{\prime}} \delta_{c^{\prime}}^{b^{\prime}}\right) \\
\langle\Sigma\rangle_{c d^{\prime}}^{a b^{\prime}} & =-\frac{v_{\mathbf{7 5}}}{4 \sqrt{6}} \delta_{c}^{a} \delta_{d^{\prime}}^{b^{\prime}}
\end{aligned}
$$

where, in the above expressions, $a, b, \cdots$ run $1-3$, while $a^{\prime}, b^{\prime}, \cdots$ run $4-5$. (Then, the $X$ - and $Y$-boson mass is $m_{X, Y}=g_{5} v_{75}$.) In this case, the effect of the $S U(5)_{\text {GUT }}$ breaking in the superpotential should be second (or higher) order in $v_{\mathbf{7 5}}$; we consider the following superpotential:

$$
\begin{aligned}
W & =\lambda_{0} S \bar{\Psi}^{\alpha} \Psi_{\alpha}+\frac{\lambda_{1}}{M_{\mathrm{Pl}}^{2}} S \bar{\Psi}^{\alpha} \Sigma_{\alpha \epsilon}^{\gamma \delta} \Sigma_{\gamma \delta}^{\beta \epsilon} \Psi_{\beta} \\
& =\lambda_{0}\left(1+\frac{1}{12} \epsilon_{\mathbf{7 5}}^{2}\right) S \bar{\psi}_{d} \psi_{d}+\lambda_{0}\left(1+\frac{1}{4} \epsilon_{\mathbf{7 5}}^{2}\right) S \bar{\psi}_{l} \psi_{l}+\cdots
\end{aligned}
$$

where

$$
\epsilon_{\mathbf{7 5}}=\frac{\lambda_{1}^{1 / 2} v_{\mathbf{7 5}}}{\lambda_{0}^{1 / 2} M_{\mathrm{Pl}}}
$$

In this case, the gaugino masses are given by

$$
M_{A}=\frac{g_{A}^{2}}{16 \pi^{2}}\left(1+\kappa_{A}^{(\mathbf{7 5})} \epsilon_{\mathbf{7 5}}^{2}\right) \Lambda
$$

with $\kappa_{1}^{(\mathbf{7 5})}=\frac{11}{60}, \kappa_{2}^{(\mathbf{7 5})}=\frac{1}{4}, \kappa_{3}^{(\mathbf{7 5})}=\frac{1}{12}$.

In Fig. 2, we show the EDMs with $\left|\epsilon_{\mathbf{7 5}}\right|=10^{-2}$. We take $\arg \left(\epsilon_{\mathbf{7 5}}\right)=\frac{\pi}{4}$, which maximizes the EDMs. In panels (a), (c) and (e), the messenger scale is taken to be $M_{\text {mess }}=10^{6} \mathrm{GeV}$, while $M_{\text {mess }}=10^{12} \mathrm{GeV}$ in (b), (d) and (f). The electron EDM is shown in the panels (a)

\footnotetext{
\#5 If the GUT symmetry is broken by a field in a real (but not adjoint) representation of $S U(5)_{\mathrm{GUT}}$, the leading order contribution of the GUT symmetry breaking is always $\left(M_{\mathrm{GUT}} / M_{\mathrm{Pl}}\right)^{2}$ as far as all the operators allowed by the $S U(5)_{\text {GUT }}$ symmetry are present. Then, in those cases, the sizes of the EDMs are of the same order of the case with the GUT symmetry breaking by a $\mathbf{7 5}$ representation.
} 


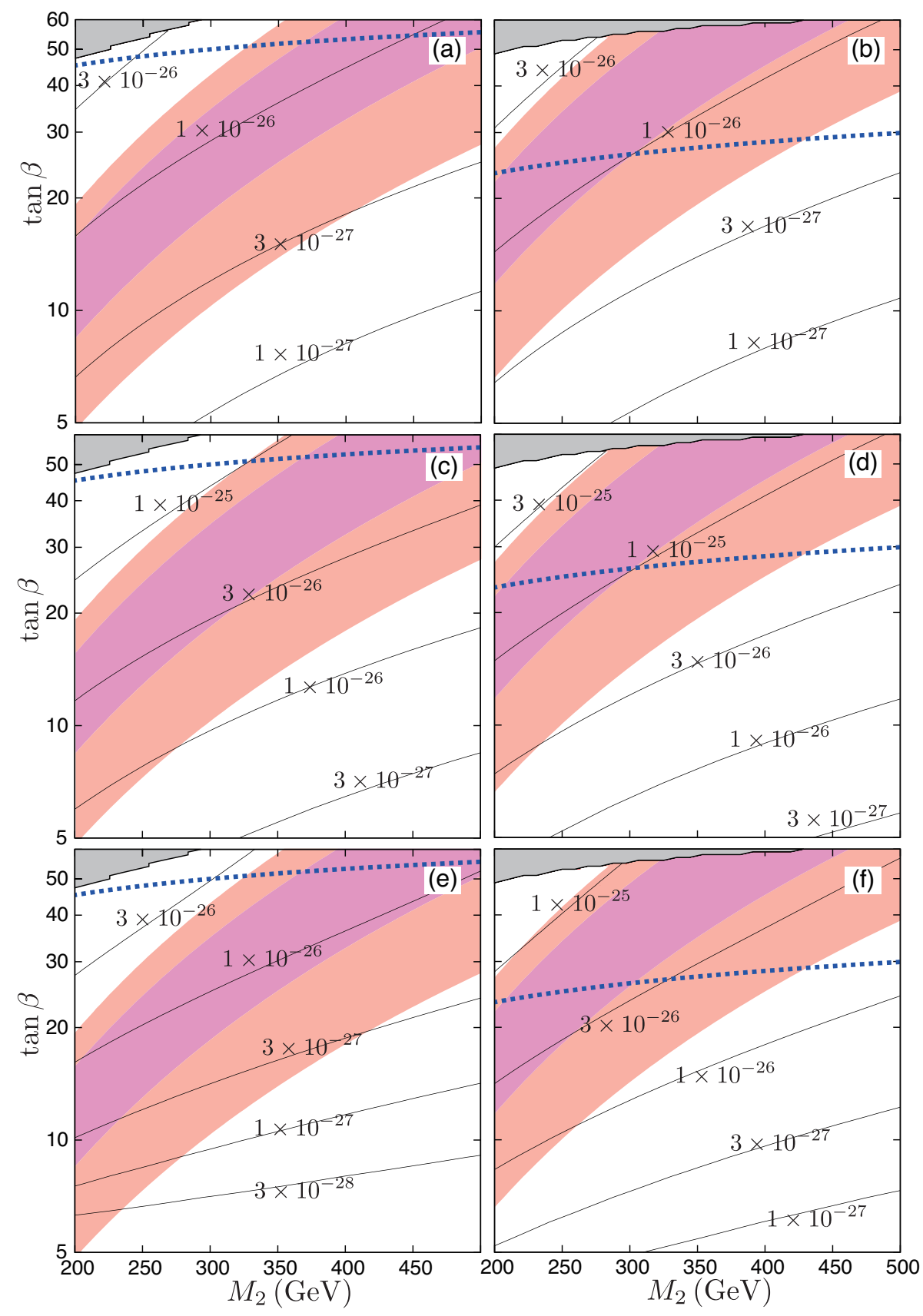

Figure 1: Contours of EDMs of the electron ((a), (b)), the neutron ((c), (d)) and $\tilde{d}_{q}((\mathrm{e}),(\mathrm{f}))$ on $M_{2}$ vs. $\tan \beta$ plane. Here, we take $\left|\epsilon_{\mathbf{2 4}}\right|=10^{-2}$, and $\arg \left(\epsilon_{\mathbf{2 4}}\right)=\frac{\pi}{2}, M_{\text {mess }}=10^{6} \mathrm{GeV}$ ((a), (c), (e)) and $10^{12} \mathrm{GeV}((\mathrm{b}),(\mathrm{d}),(\mathrm{f}))$. The sign of $\mu_{H}$ is positive. Blue dotted lines correspond to $B_{\mu}\left(M_{\text {mess }}\right)=0$. In the pink (orange) regions, the muon MDM becomes consistent with the experimental value at $1 \sigma(2 \sigma)$ level. The gray regions are excluded due to un-successful electroweak symmetry breaking. The numbers in the figures are EDMs in units of $e \mathrm{~cm}((\mathrm{a})-(\mathrm{d}))$ or $\tilde{d}_{q}$ in units of $\mathrm{cm}((\mathrm{e})$ and $(\mathrm{f}))$. 
and (b), while neutron EDM are shown in panels (c) and (d). In addition, the results of $\tilde{d}_{q}$ are shown in panel, (e) and (f). As one can expect, the EDM constraints are less severe compared to the case with $\mathbf{2 4}$ representation. This indicates that the EDM constraints can be avoided if operators linear in the $G_{\mathrm{GUT}}$ breaking field are somehow forbidden. However, such models are testable if the experimental sensitivities on the EDMs are improved by two orders of magnitude.

Before closing this section, we comment on another effect, i.e., renormalization group effect, which modifies the GUT relation among the coupling constants. The renormalization group equations of $\lambda_{i j}^{(Q)}$ and $M_{i j}^{(Q)}$ are given by

$$
\begin{aligned}
\frac{d \lambda_{i j}^{(Q)}}{d \ln \mu} & =\gamma_{i k}^{(\bar{Q})} \lambda_{k j}^{(Q)}+\gamma_{j k}^{(Q)} \lambda_{i k}^{(Q)}+\gamma^{(S)} \lambda_{i j}^{(Q)}, \\
\frac{d M_{i j}^{(Q)}}{d \ln \mu} & =\gamma_{i k}^{(\bar{Q})} M_{k j}^{(Q)}+\gamma_{j k}^{(Q)} M_{i k}^{(Q)},
\end{aligned}
$$

where $\gamma^{(X)} \equiv-\frac{1}{2} \frac{\partial \ln Z_{X}}{\partial \ln \mu}$ (with $Z^{(X)}$ being the wave-function renormalization factor) is the anomalous dimension of the chiral superfield $X$. Then, the solutions of the above equations are given by

$$
\begin{aligned}
\lambda_{i j}^{(Q)}(\mu) & =\zeta_{i k}^{(\bar{Q})}(\mu) \zeta_{j l}^{(Q)}(\mu) \zeta^{(S)}(\mu) \lambda_{k l}^{(Q)}\left(M_{\mathrm{GUT}}\right) \\
M_{i j}^{(Q)}(\mu) & =\zeta_{i k}^{(\bar{Q})}(\mu) \zeta_{j l}^{(Q)}(\mu) M_{k l}^{(Q)}\left(M_{\mathrm{GUT}}\right)
\end{aligned}
$$

where

$$
\zeta_{i j}^{(Q)}=\delta_{i j}+\sum_{n=1}^{\infty} \int_{\ln M_{\mathrm{GUT}}}^{\ln \mu} d t_{1} \int_{\ln M_{\mathrm{GUT}}}^{t_{1}} d t_{2} \cdots \int_{\ln M_{\mathrm{GUT}}}^{t_{n-1}} d t_{n} \gamma_{i k_{1}}^{(Q)}\left(t_{1}\right) \gamma_{k_{1} k_{2}}^{(Q)}\left(t_{2}\right) \cdots \gamma_{k_{n-1} j}^{(Q)}\left(t_{n}\right)
$$

and

$$
\zeta^{(S)}=\exp \left[\int_{\ln M_{\mathrm{GUT}}}^{\ln \mu} d t \gamma^{(S)}(t)\right]
$$

with $t=\ln \mu$. A similar expression holds for $\zeta_{i k}^{(\bar{Q})}$. Then, for multiplets $Q$ and $Q^{\prime}$ originating from the same multiplet of $G_{\mathrm{GUT}}$, the equality $\Lambda^{(Q)}=\Lambda^{\left(Q^{\prime}\right)}$ is realized if $\lambda_{i j}^{(Q)}=\lambda_{i j}^{\left(Q^{\prime}\right)}$ and $M_{i j}^{(Q)}=M_{i j}^{\left(Q^{\prime}\right)}$ at the GUT scale. Using the fact that $\sum_{Q} c_{A}^{(Q)}$ become independent of the standard-model gauge group (i.e., $A=S U(3)_{\mathrm{C}}, S U(2)_{\mathrm{L}}$, and $U(1)_{\mathrm{Y}}$ ) as far as all the fields in the same multiplet of $G_{\mathrm{GUT}}$ are contained in the summation, $m_{A} / g_{A}^{2}$ becomes universal and hence there is no relative phase among gaugino masses.

\section{Effect of Supergravity}

Next, we consider the phases from the supergravity effect. When the standard model is supersymmetrized, naturally we consider local supersymmetry, i.e., supergravity. Then, supergravity effect may also induce small but non-negligible CP violating phases. 


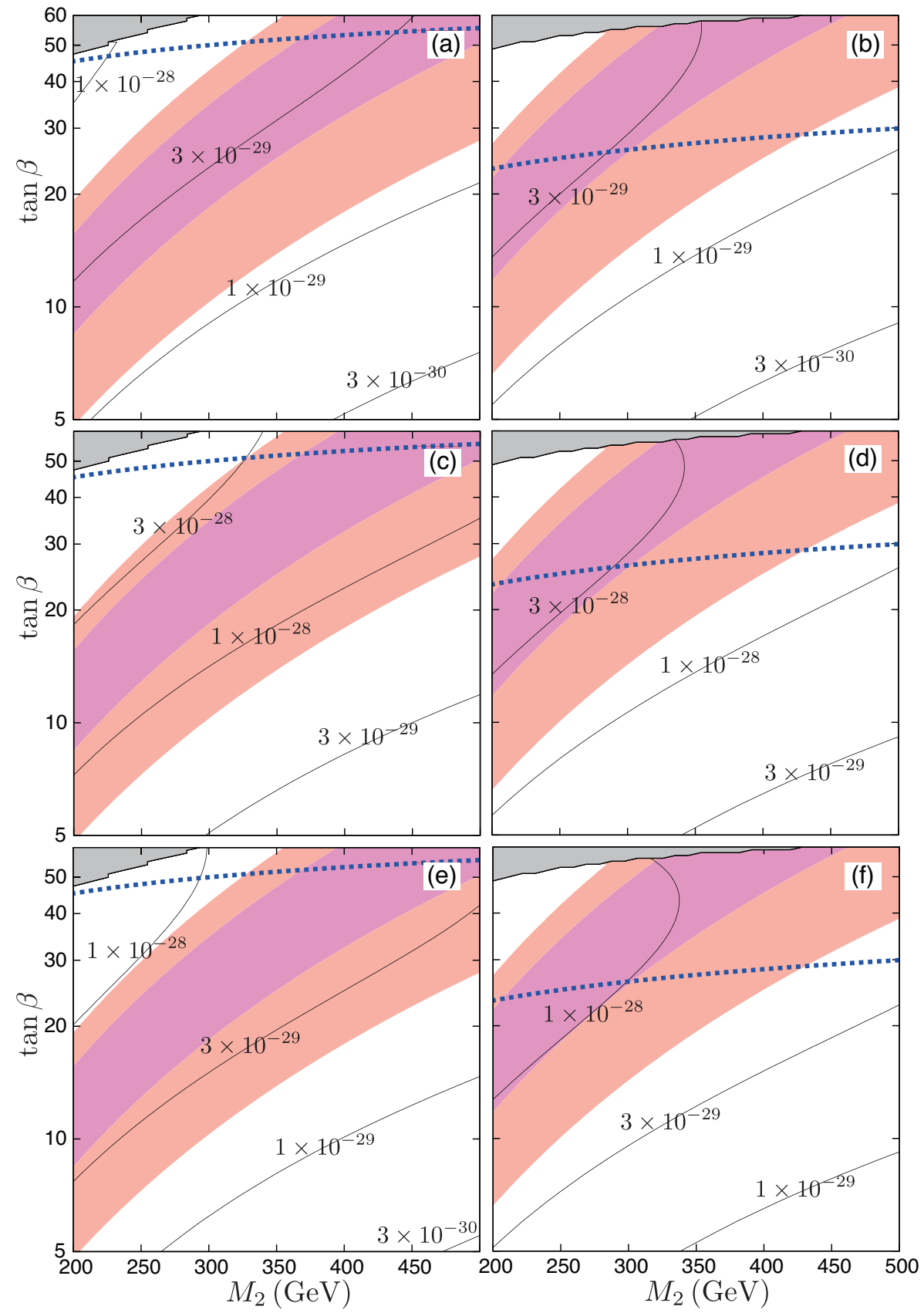

Figure 2: Same as Fig. 1, but with $\left|\epsilon_{\mathbf{7 5}}\right|=10^{-2}$ and $\arg \left(\epsilon_{\mathbf{7 5}}\right)=\frac{\pi}{4}\left(\right.$ and $\left.\epsilon_{\mathbf{2 4}}=0\right)$. 
Concerning the phases $\phi_{A}$, one should consider the effect on the $B_{\mu}$ parameter \#6 Naturally, such an effect is estimated to be of the order of the gravitino mass $m_{3 / 2}$. Importantly, in general, the supergravity contribution to $B_{\mu}$ is complex, and its phase is not aligned to those of gaugino masses. Thus, this becomes a new source of $\mathrm{CP}$ violation.

To study its consequence, we calculate the EDMs taking account of the supergravity effects on the phase in $B_{\mu}$ parameter. Here, we parameterize $B_{\mu}$ as

$$
B_{\mu}=B_{\mu}^{(0)}+B_{\mu}^{(\mathrm{SUGRA})},
$$

where $B_{\mu}^{(\mathrm{SUGRA})}$ is the supergravity contribution to the $B_{\mu}$ parameter, which is expected to be of the order of the gravitino mass. Because we are interested in the gauge mediation model, the gravitino mass is much smaller than the electroweak scale, and hence $\left|B_{\mu}^{\text {(SUGRA) }}\right| \ll\left|B_{\mu}\right|$. As mentioned before, we do not specify the source of the dominant contribution $B_{\mu}^{(0)}$; we assume that $\phi_{A} \rightarrow 0$ as $B_{\mu}^{(\mathrm{SUGRA})} \rightarrow 0$ to derive a conservative constraint. In addition, we adopt the usual GUT relation among the gaugino masses; the $\epsilon_{\mathbf{2 4}}$ and $\epsilon_{\mathbf{7 5}}$ parameters used in the previous section are set to be zero.

We calculate EDMs taking $\left|B_{\mu}^{(\text {SUGRA })}\right|=100 \mathrm{MeV}$ and $\arg \left(B_{\mu}^{\text {(SUGRA) }}\right)=\frac{\pi}{2}$. With this choice, the phase $\phi_{A}$ is of the order of $10^{-3}$. The contours of constant EDMs are shown in Fig. 3. One can see that, the results are not sensitive to the messenger scale in the present set up in which the messenger scale is not related to the gravtino mass. In the parameter region which is consistent with the constraint (13), the electron and mercury EDMs become marginally consistent with the experimental constraints when the gravitino mass is $\sim 100 \mathrm{MeV}$. Notice that the supergravity contributions to the off-diagonal elements of the sfermion mass matrices are typically $\sim m_{3 / 2}^{2}$. Thus, the $\mathrm{CP}$ violations may put severer upper bound on the gravitino mass than the flavor violations.

In the present framework, the EDMs are proportional to $\operatorname{Im}\left(B_{\mu}^{(\text {SUGRA })}\right)$ as far as $\left|B_{\mu}^{(\text {SUGRA })}\right|$ is much smaller than $\left|B_{\mu}\right|$. Because $\left|B_{\mu}^{(\mathrm{SUGRA})}\right|$ is expected to be of the order of the gravitino mass $m_{3 / 2}$, we can see that gauge mediation models with $m_{3 / 2} \gtrsim 100 \mathrm{MeV}$ require some mechanism to suppress the phase in $B_{\mu}^{(\text {SUGRA) }}$ parameter (and hence that in the gravitino mass) relative to the gaugino masses if we take the anomaly in the muon MDM seriously.

\section{Summary}

In this Letter, we have studied the SUSY CP problem in gauge mediation model. We have paid particular attention to the effects of GUT symmetry breaking and the supergravity effect. Both of these effects possibly induce too large CP violating phase in the MSSM parameters to be consistent with the experimental constraints on EDMs. It was considered

\footnotetext{
\#6 $A$ parameters are also generated by the supergravity effect, and are also of the order of the gravitino mass. The effect of the phases in the $A$ parameters are at most of the same order of the that in $B_{\mu}$ because the contribution of the $A$ parameter does not have the $\tan \beta$ enhancement.
} 

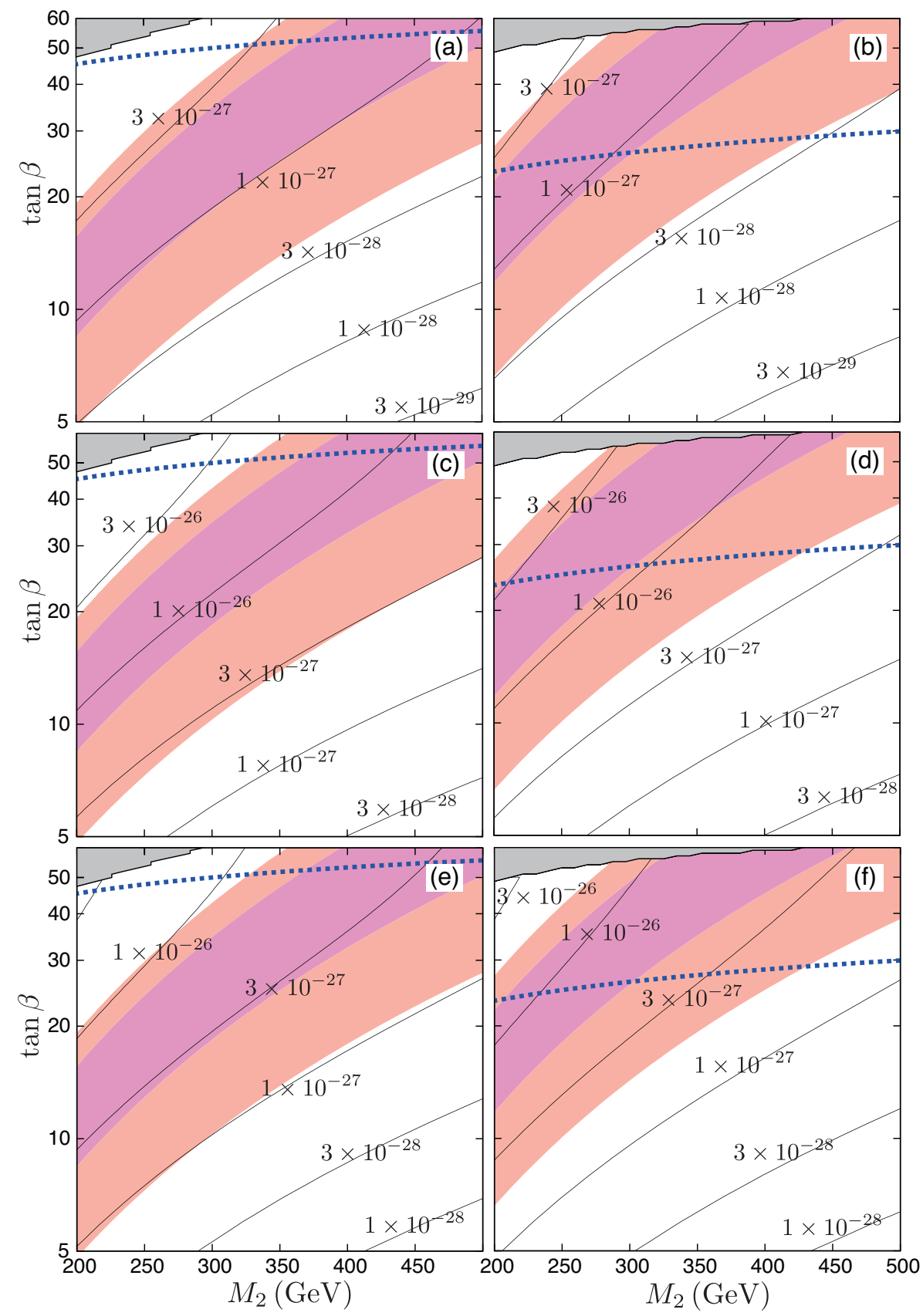

Figure 3: Same as Fig. 1, but with $\left|B_{\mu}^{\mathrm{SUGRA}}\right|=100 \mathrm{MeV}$ and $\arg \left(B_{\mu}^{\mathrm{SUGRA}}\right)=\frac{\pi}{2}\left(\right.$ and $\epsilon_{\mathbf{2 4}}=\epsilon_{\mathbf{7 5}}=$ $0)$. 
that the SUSY CP problem can be avoided in the gauge mediation model in which $B_{\mu}$ parameter is not generated at the messenger scale. However, the $\mathrm{CP}$ violating phases discussed in this letter cannot be eliminated even in such a model.

In particular, the effect of the GUT symmetry breaking spoils the GUT relation among the gaugino masses if a mass term is introduced to the vector-like messenger multiplets; consequently, the phases of the gaugino masses may become different. In the case that operators which are linear in $G_{\mathrm{GUT}}$ breaking field are allowed, the EDMs are likely to be too large in the parameter region where the SUSY contribution to the muon MDM explains the discrepancy between the experimental and standard-model values. In other cases, the EDMs are expected to be smaller than the current experimental bounds. However, even so, the effects may be observed if the experimental sensitivity to the EDMs can be improved by two orders of magnitude.

\section{Acknowledgements}

The authors thank R. Sato and K. Yonekura for useful discussion. The work of T.M. is supported by Grant-in-Aid for Scientific resear ch from the Ministry of Education, Science, Sports, and Culture (MEXT), Japan, No. 22540263 and No. 22244021, and the work of N.Y. is supported by Grand-in-Aid for Scientific Research, No.22-7585 from JSPS, Japan.

\section{References}

[1] See, for example, F. Gabbiani, E. Gabrielli, A. Masiero and L. Silvestrini, Nucl. Phys. B 477, 321 (1996).

[2] M. Dine, A. E. Nelson, Phys. Rev. D48, 1277 (1993).

[3] M. Dine, A. E. Nelson, Y. Shirman, Phys. Rev. D 51, 1362 (1995).

[4] M. Dine, A. E. Nelson, Y. Nir, Y. Shirman, Phys. Rev. D 53, 2658 (1996).

[5] M. Dine, W. Fischler and M. Srednicki, Nucl. Phys. B 189, 575 (1981).

[6] S. Dimopoulos and S. Raby, Nucl. Phys. B 192, 353 (1981).

[7] M. Dine and W. Fischler, Phys. Lett. B 110, 227 (1982).

[8] C. R. Nappi and B. A. Ovrut, Phys. Lett. B 113, 175 (1982).

[9] L. Alvarez-Gaume, M. Claudson and M. B. Wise, Nucl. Phys. B 207, 96 (1982).

[10] T. Moroi, Phys. Lett. B447, 75 (1999).

[11] R. Rattazzi, U. Sarid, Nucl. Phys. B501 , 297 (1997). 
[12] E. Gabrielli, U. Sarid, Phys. Rev. Lett. 79 , 4752 (1997).

[13] K. A. Intriligator, N. Seiberg and D. Shih, JHEP 0604, 021 (2006).

[14] For review, see, for example, R. Kitano, H. Ooguri and Y. Ookouchi, arXiv:1001.4535 [hep-th].

[15] H. Murayama and Y. Nomura, Phys. Rev. Lett. 98, 151803 (2007).

[16] H. Murayama and Y. Nomura, Phys. Rev. D 75, 095011 (2007).

[17] S. Dimopoulos, H. Georgi, Nucl. Phys. B193 , 150 (1981).

[18] N. Sakai, Z. Phys. C11 , 153 (1981).

[19] K. Nakamura et al. [Particle Data Group], J. Phys. G 37, 075021 (2010).

[20] G. -C. Cho, K. Hagiwara, Y. Matsumoto, D. Nomura, arXiv:1104.1769 [hep-ph].

[21] M. Pospelov, A. Ritz, Phys. Rev. Lett. 83, 2526 (1999).

[22] M. Pospelov, A. Ritz, Phys. Rev. D63, 073015 (2001).

[23] J. Hisano, Y. Shimizu, Phys. Rev. D 70, 093001 (2004).

[24] W. C. Griffith, M. D. Swallows, T. H. Loftus, M. V. Romalis, B. R. Heckel, E. N. Fortson, Phys. Rev. Lett. 102, 101601 (2009).

[25] M. Pospelov, A. Ritz, Annals Phys. 318, 119 (2005).

[26] J. R. Ellis, J. S. Lee, A. Pilaftsis, JHEP 0810, 049 (2008).

[27] A. Masiero, D. V. Nanopoulos, K. Tamvakis, T. Yanagida, Phys. Lett. B115, 380 (1982).

[28] B. Grinstein, Nucl. Phys. B206 , 387 (1982). 